\title{
Design and Realization of Automatic Forming Machine for Welding Rod Box Based on PLC Control
}

\author{
Bing Wang \\ Information Office \\ University of Shanghai for Science and Technology \\ Shanghai, China \\ wbing73@usst.edu.cn
}

\begin{abstract}
The device controls the rotation of the servo motor by PLC, so as to control to the movement of the feeder device, making a coordination work with paper feeder, paper to transfer machine, box forming machine and the paper fetching machine. This device achieved a highly automation of forming the welding rod box, solved the inefficiency problem of the current factory electrode box molding.
\end{abstract}

Keywords-PLC; welding rod box; forming machine; automation

\section{INTRODUCTION}

Currently, all electrode boxes are assembled manually, then workers put the electrode box forming in ultrasonic welding machine for welding. In this way, not only the human cost, the efficiency is relatively low[1]. Our electrode box automatic molding machine is composed of a paper machine, paper machine, box forming machine and paper machine. Paper feeder will put the electrode box with indentation on molding gloves in paper transfer machine. The paper will transfer with paper transfer machine to the bottom of the box forming machine. In this way, the box forming machine is able to form the paper. Formed electrode box will transfer to the bottom of the paper taking machine, so that the paper fetching machine is able to taking the molding paper out, and then put in the specified location. The device will realize the automatization of forming the electrode box. The total of the whole machine is realized by using PLC (Programmable Logic Controller)for rotation of the servo motor [2][3].

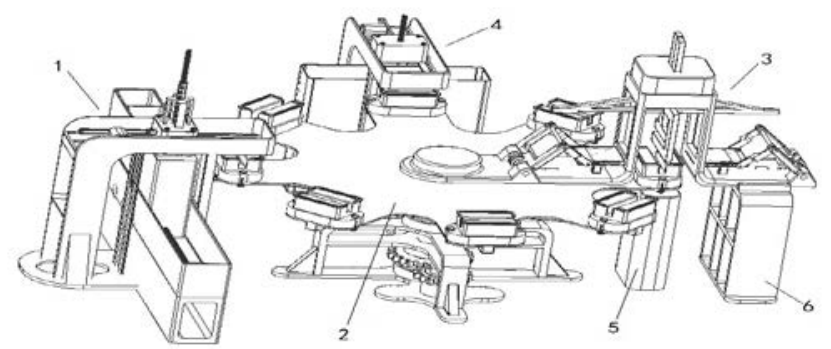

Fig. 1. The overall structure of the Electrode box automatic molding machine

(1. paper feeder 2. paper transfer machine 3. box forming machine 4. paper taking machine 5 . support frame 16 . support frame 2 )

\author{
Shunli Wang \\ College of mechanical engineering \\ University of Shanghai for Science and Technology \\ Shanghai, China \\ wsl168168168@163.com
}

\section{OVERALL DESIGN}

Electrode box automatic molding machine is composed of a paper machine, pass paper machine, box forming machine and catch paper machine, paper machine by PLC, controls servo motor to rotate, so that paper feeder slider crank mechanism on the electrode forming groove box paper indentation on the transfer paper machine, paper machine controls PLC the rotation of servo motor, put paper on paper machine driven by the movement to just below the box forming machine, PLC controls box forming machine of the servo motor to rotate, there by forming pressure on paper box machine, box forming machine for paper molding processing, PLC controls the rotation of paper machine servo motor electrode box forming on paper machine driven by the movement to just below the paper fetching machine, PLC controls paper machine servo motor to rotate, so as to take the paper machine through the slider crank mechanism to molding paper out, into the designated location, realizing the automatic forming electrode box[4].

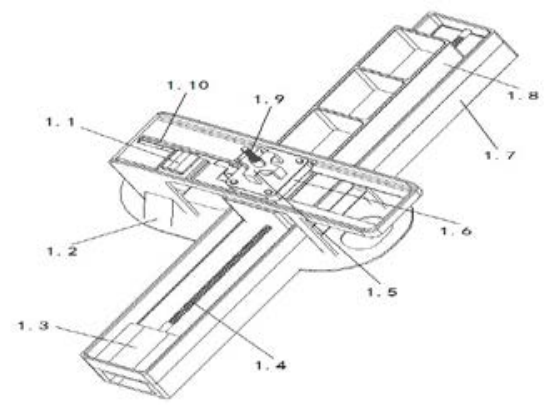

Fig. 2. The overall structure of the paper machine.

(1.1 servo motor 11.2 support frame 31.3 servo motor 21.4 screw 11.5 support frame d 1.6 car 11.7 saddle 1.8 car 21.9 a suction device for screw 1 1.10 rod piece 1 )

\section{WORKING PRINCIPLE}

Paper feeder contains servo motor, supporting frame, car seat, and screw rods, PLC controls the rotation of servo motor 2, and then rotates the screw 1 , screw 1 drives a car 2 in the seat to move back and forth, the paper of electrode box with indentations is put in the blank space of car 2. Servo motor, rod a car and a composition of a slider crank 
mechanism, PLC controls the rotation of servo motor 1 , servo motor 1 drives a crank, crank connecting rod drives the connecting rod to drive the car in a mobile and moving on support frame 3, support frame 4 is above the car, it is used to support a suction device for screw, the upper side of the screw suction device is a ball screw, the lower is the sucker, sucker is used to draw the box paper with indentation electrode, through the PLC control of the car in a servo motor is transferred, so as to drive the screw a screw suction device for drop sucker installation a pressure sensor. When the pressure of the pressure sensor reaches a certain value, PLC controls servo motor in a car will stop, at this time, chuck starts work and draws paper. After that, PLC controls car 1 within the servo motor to drive in a reversing direction. and raise the suction device for a screw up. When it rises to the original position, PLC stops the servo motor in car 1 . And the servo motor control is transferred by PLC, putting the paper onto the slider crank mechanism is formed in the box above the paper machine, PLC controls servo motor stalls, servo motor PLC controls in a small car turns, make the paper down when a fall in a certain position, chuck, spit out the paper, the PLC controls of the car in a servo motor reversal, back to the original position, PLC controls servo motor in a car stopped, and then the PLC controls servo motor through a reverse crank slider. The sucker to car just above the second, PLC controls servo motor to stop rotating, so the cycle, in order to achieve the screw sucker device of electrode box paper absorbing and storing, screw chuck system from the second car compartment of the lattice draw with indentation of the electrode box paper, by a slider crank mechanism driven mobile car, put the paper into the molding groove of the conveyor[5].

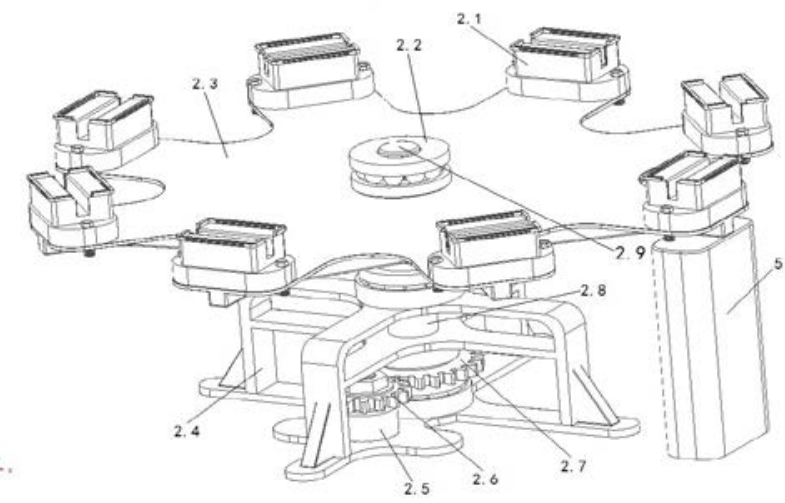

Fig. 3. The overall structure of the pass paper machine.

(2.1 forming groove 2.2 thrust bearing 2.3 turntable 2.4 support frame 52.5 servo motor 32.6 gear 12.7 gear 22.8 sleeve 2.9 spindle)

Transfer paper machine contains forming groove, thrust bearing, turntable, support frame, servo motor, composed of gear and a sleeve. PLC controls servo motor 3 and stop rotating, the servo motor 3 drives the gear shaft 3 through a rotation gear and a gear meshing gear drive, rotation, gear and spindle through the key connected together, so as to drive the rotation of the spindle, the spindle and the rotary disc are connected together, so as to drive the rotating disk to rotate. The molding groove is fixed on the rotary disc through a screw and nut, rotate and forming groove in a rotating disk drive, so as to realize the just below the electrode box from the motion to the box forming machine and paper machine take the paper machine. The rotating disc is connected with the main shaft together on the underside of the rotating disc through the thrust bearing and other parts connected to upper bearing and box forming machine through the support frame is connected with the lower bearing supporting frame 5 connected by thrust, support the bracket five is divided into three layers. The first layer and the two layers are connected through a sleeve which is sheathed on the main shaft, and the two layer and the bottom layer are connected through the thrust bearing and the gear.

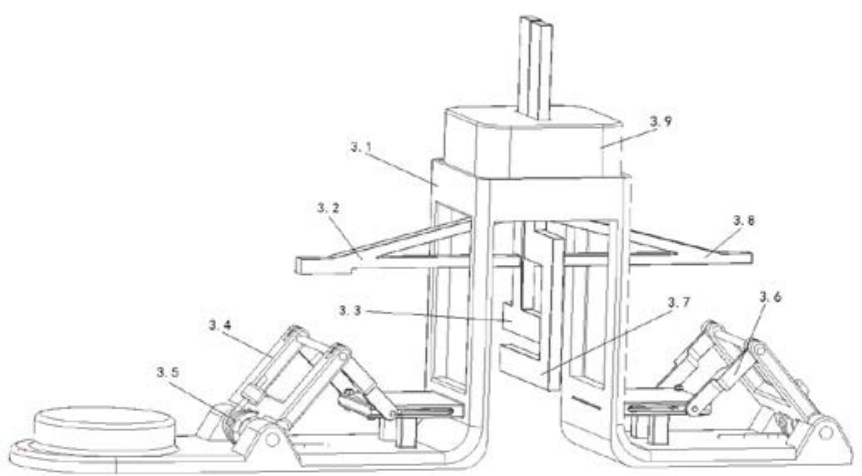

Fig. 4. The overall structure of the press paper machine.

(3.1 support frame 63.2 pressure lever 13.3 pressure lever 23.4 crank 3.5 spring 3.6 connecting rod 3.7 sleeper 3.8 ultrasonic welding apparatus 3.9 motor control box)

Box forming machine is composed of a support frame, pressure lever, crank, spring, connecting rod, sleeper, ultrasonic welding apparatus, servo motor, push plate, slider and block a box with the disk electrode indentation has five sides, when it is formed, two faces of it are overlapped together, when the paper transfer machine carried the paper to the right position where below the press paper machine, PLC controls the two servo motor turned oppositely, which driven sleepers and ultrasonic welding apparatus moved up and down. Firstly, PLC controls servo motor 4 clockwise, which made the sleeper fell, which made the indentation paper pressed into the molding groove. At the same time, the three surfaces of the box have connected the three electrode surfaces of the forming groove tightly, only one surface is in the open state, at this time, PLC controls servo motor 5 clockwise, which made the ultrasonic welding apparatus fall. At the same time, connected with the pressure rod welding apparatus in ultrasonic and pressure above a rod two with ultrasonic welding machine is getting down, due to a pressure lever at the bottom than the bottom part of the convex pressure lever 2. So in the process of decline, a pressure lever 1 to come into contact with the slider crank mechanism than the pressure lever 2 . When pressure lever 1 pressure to the shaft will drive the movement of slider crank mechanism is rotated, spring compression that slide in a slide block slot, thereby promoting pushing inwards. While pushing inward movement will push through the slot to come into contact with the upper electrode box so as to erect a piece of paper in a pressure lever pressing, falling into the push shaft piece inward movement at the same time, pressure lever 2 the hit on the right side of the shaft causes the push piece by pushing slot inwards into contact 
with the upper electrode box erected on the other side of the paper, due to a pressure lever and a pressure rod to the shaft of the two contact time is different. So it will not lead to the upper electrode on both sides of the paper box erected cross. The two stands on the side of the box surface electrode the overlap is flattened, in the two plane[6]. Most side overlap flattened at the same time, instrument has fallen to the upper surface of the electrode box, the ultrasonic welding, PLC controls servo motor 5 stops, ultrasonic welding apparatus work by making paper vibration, friction heat to make the paper together, when the paper together, PLC servo motor 5 while the rise in ultrasonic welding apparatus, a pressure lever and a pressure rod two with ultrasonic welding equipment to rise. At this time, in the spring under the action of the slider crank drive the slider and pushing outward, thus form the electrode box left, until hitting the most lateral slide block. When the ultrasonic welding apparatus up to the original position, PLC controls servo motor 5 stops, the PLC controls servo motor 3 To rotate, so that the paper machine rotation, due to the convex groove forming one end of the baffle, baffle and the other end is not convex, so that paper machine at the same time, baffle take down the forming of the electrode box from the sleepers, in groove shape. When forming the electrode box success from sleepers, PLC controls servo motor 4 reversal, so that the sleeper began to rise, rise to the original location, so a welding rod box molding step is complete, processing good caddy in the biography of paper machine drive movement to just below the paper fetching machine.

The crank slider mechanism consisting of a spring, a crank, a connecting rod and a sliding block is driven to drive the push plate to move around, and the stop block controls the limit position of the outer movement of the sliding block, and the pressure lever controls the limit position of the inward movement of the push piece.

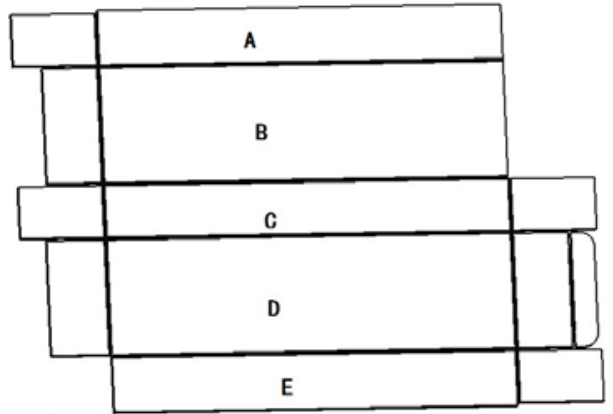

Fig. 5. The overall structure of the welding rod box paper with indentation

Sleeper is longer than molding groove, sleeper's width is little smaller than the width of the groove, the width of the molding groove is as same as the electrode box, the length of the electrode box slightly less than the length of the molding groove, and groove forming the end of the have end width with the paper pressing machine sleeper width but then the width of the electrode box small opening, is provided with a convex baffle. The same size of the width of the opening of the other end of the size and the caddy, no baffle is, so as to ensure the welding rod box after the completion of welding, functional paper smoothly the electrode box away.
PLC controls servo motor 3 rotate, therefore the paper machine welding rod paper with indentation to paper box under pressure box machine, PLC controls servo motor 3 stop rotating, so that the paper machine stops running, the sleepers dropping which box paper with electrode indentation forming groove in the bottom forming the paper box groove and the electrode area $C$ with indentation contact area with $C$ electrode box paper indentation contact with the sleeper bottom, the left side of the molding groove contact with the electrode box paper $\mathrm{D}$ with indentation, indentation with electrode box paper D District contact with the sleepers left side, right side in contact with the electrode forming slot paper box B area with indentation, indentation with electrode contact with B paper box sleeper right, and then under the servo motor five under the action of the instrument down the ultrasonic welding. The crank slider mechanism is composed of a spring and the push rod piece moves inward The paper, pushing $A$ and $E$ area electrode box with indentation flattening, let $\mathrm{A}$ and $\mathrm{E}$ coincide, at this time, the ultrasonic welding meter just down to $\mathrm{A}$ and $\mathrm{E}$ regions of overlapping area, instrument of the welding head on the vibration friction heat to $\mathrm{A}$ and $\mathrm{E}$ together by ultrasonic welding, forming an area, AE area, the electrode box welding, ultrasonic welding equipment up, back to the position. At the same time, push back the PLC control servo motor 3 to transfer paper machine in the molding groove at one end baffle under the action of promoting the good welding molding box forward, and get out of the box from the sleeper. When the carton completely out of sleeper, sleeper through the motor box back to the original position, then a complete end electrode box molding operation, then repeat the operation[7].

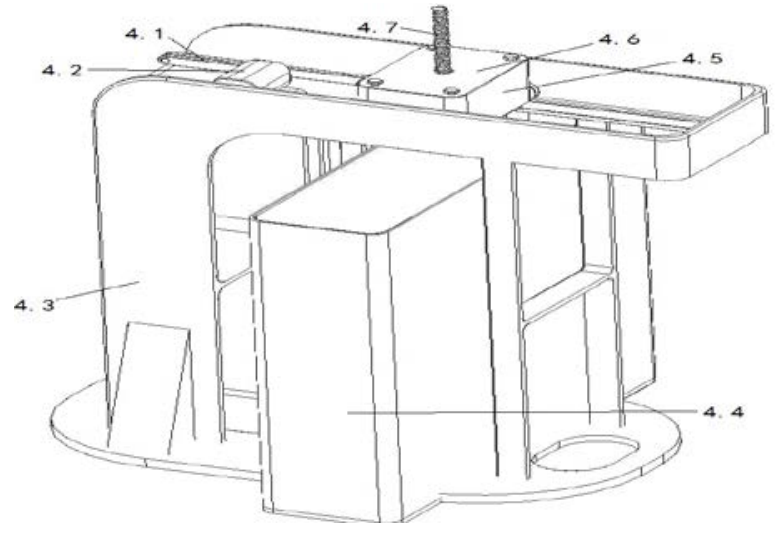

Fig. 6. The overall structure of the take paper machine.

(4.1 rod piece 24.2 servo motor 64.3 support frame 84.4 box 4.5 car 34.6 car 44.7 a suction device for screw 2)

The paper machine was consisted of rod servo motor, support frame, car and screw suction device. The servo motor, rod two and a car made up a trolley crank slider system. When the paper transform machine carried the finished electrode to position where just below the paper fetching machine, PLC controls servo motor 6 turned clockwise, so that the servo motor 6 drives the crank move which lies in the rod piece 2 . And the crank drives the connecting rod moving rods of the second connecting rod to drive the car 3 in support 8 movement, movement to just above the electrode box forming the car 3 in the servo drive, PLC servo motor to controls the in 
car 3 forward, drive motion of screw suction device 3, so that the screw suction device down to the electrode box forming position, through the pressure sensor, PLC servo motor to controls the car 3 stops, the chuck suction electrode box, PLC controls the servo motor reversal in car 3 , and then this sucker device of screw up. When the screw chuck device rises to a certain position, PLC controls servo motor stops rotating in the car 3, and servo motor 4 rotation, through the crank slider mechanism make car 3 moves outward, moved to is at the top of the storage boxes, at this time, the chuck spit out the forming electrode box. And the forming electrode box are collected in storage box.

\section{SUMMARY}

The automatic electrode box forming equipment combined the paper feeder, paper transfer machine and box forming machine together cleverly. It highly automates the production of electrode box. And solve the current problem about the inefficiency of production in factory. At the same time, it not only saved the cost and human resources, but also increased the productivity of electrode box dramatically.

\section{REFERENCES}

[1] Sun Heng, Chen zuomo.Theory of machines and mechanisms[C].Higher Education Press,2001. (In Chinese)

[2] DALLAS Semiconductor.Automatic Identification Data Book[J]. Journal of Women's Health .

[3] Baojiang Sun,Honglei Qin,Shituan Shen.An Automatioc Design Technique for Hardware System of ATS[J]. Systems Readiness Technology Conference . 2006: 386-396.

[4] F. Gu,Y. Shao,N. Huc,et al.Electrical motor current signal analysis using a modified bispectrum for fault diagnosis of downstrea mechanical equipment[J]. Mechanical Systems and SignalProcessiig. 2011,25(1):360-372.

[5] Achmad Widodo,Bosuk Yang.Support vector machine in machine condition monitoring and fault diagnosis[J]. Journal of Mechanical Systems. Vol.21, August 2007, P 2560-2574.

[6] Peng Chen,Toshio Toyota,Zhengja He.Automated function generation of symptom parameters and application to fault diagnosis of machinery under variable operating conditions[J]. IEEE Transactions on systems, man, and cybernetics- Part A: Systems and Humans . 2001, 31(6):775781.

[7] ROBERT X G,YAN R Q.Wavelets:Theory andapplications for manufacturing[J]. Springer Ebooks, 2010. 